\title{
Des fonctions de la simulation des situations de travail en ergonomie
}

\section{François Daniellou}

\section{(2) OpenEdition \\ 12 Journals}

Édition électronique

URL : http://journals.openedition.org/activites/1696

DOI : 10.4000/activites. 1696

ISSN : 1765-2723

Éditeur

ARPACT - Association Recherches et Pratiques sur les ACTivités

Référence électronique

François Daniellou, «Des fonctions de la simulation des situations de travail en ergonomie », Activités [En ligne], 4-2 I octobre 2007, mis en ligne le 15 octobre 2007, consulté le 30 avril 2019. URL : http:// journals.openedition.org/activites/1696; DOI : 10.4000/activites.1696

\section{(ब) $(\oplus \Theta$}

Activités est mis à disposition selon les termes de la licence Creative Commons Attribution - Pas d'Utilisation Commerciale - Pas de Modification 4.0 International. 


\title{
Des fonctions de la simulation des situations de travail en ergonomie
}

\author{
François Daniellou \\ Département d'Ergonomie, Institut de Cognitique, Université Victor Segalen Bordeaux 2 \\ Case 55, 146 rue Léo-Saignat, F33076 BORDEAUX Cedex \\ francois.daniellou@idc.u-bordeaux2.fr
}

\begin{abstract}
Ergonomic simulations may be classified according to the role that they assign to the future users, which may either be modelled in technical tools, subjects of controlled experiments, or participants in a participatory process. In the latter case, simulations may produce more than technical improvements. They may result in new developments of individual and collective activity.
\end{abstract}

KEYWORDS

Simulation, participatory ergonomics, theory of practice.

\section{1.- Simuler pour ou avec les utilisateurs?}

Des simulations de postes de travail sont utilisées dans de nombreux contextes industriels ou tertiaires, avec des objectifs divers. Trois utilisations principales sont: i) l'anticipation, en phase de conception, de certaines caractéristiques de l'activité future des usagers, afin de diagnostiquer des problèmes éventuels; ii) l'analyse des processus cognitifs dans des situations qu'il est difficile de provoquer ou d'observer dans la réalité; iii) la formation des travailleurs (Pastré, 2005).

Les termes «simulation ergonomique » sont devenus banals, et leur recherche sur internet révèle des centaines de propositions commerciales. Pour autant, cette généralisation masque probablement des différences fondamentales entre les usages des simulations ergonomiques dans les processus de conception.

Une façon de catégoriser les différentes approches de la simulation des situations de travail est d'identifier le statut des utilisateurs ${ }^{1}$ dans le déroulement de la simulation:

- a) dans bien des cas, les utilisateurs ne sont pas physiquement présents, ils sont modélisés par des dispositifs techniques comme un mannequin, un programme informatique, etc., disponibles dans les outils de conception que les concepteurs utilisent sans l'aide d'un ergonome;

- b) dans d'autres cas, des utilisateurs ou des sujets censés ressembler aux utilisateurs participent à une expérimentation contrôlée, leurs comportements sont analysés par un expert ergonome, qui les interrogera - ou non - sur leur activité et leur perception du système simulé et de son utilisabilité;

— c) enfin, les utilisateurs peuvent être invités à prendre part à une démarche ergonomique participative, où la simulation est l'un des éléments d'une implication plus globale des différents acteurs dans le processus de conception (Haslegrave, Wilson, \& Corlett, 1990; Wilson, 1991).

1. L'objet de cet article n'est pas de discuter la pertinence du concept d'« utilisateur ». On désignera par ce terme les opérateurs qui travailleront, à quelque titre que ce soit, dans les situations de travail considérées. 
Cette catégorisation est différente de celle qu'on pourrait construire en se basant sur les dispositifs techniques utilisés pour la simulation: par exemple, la réalité virtuelle peut être utilisée: a) par une équipe de conception qui ne fait appel à aucun utilisateur, b) par un ergonome pour mettre en place une expérimentation contrôlée, ou c) dans le cadre d'un groupe de travail rassemblant différents acteurs pour instruire les choix de conception.

\section{1.-Intérêts et limites des simulations par un expert}

Les simulations effectuées par des concepteurs ou des ergonomes, à l'aide de moyens techniques, sans la présence réelle des utilisateurs, supposent une triple modélisation: un modèle de l'être humain, un modèle du système de travail, et un modèle du travail à réaliser.

Le modèle de l'être humain est habituellement un modèle anthropométrique plus ou moins sophistiqué. Dans quelques cas, des données biomécaniques sont intégrées, comme la prise en compte des angles ou des efforts. Des dimensions perceptives peuvent également être incluses (visibilité, par exemple).

Les modèles du système de travail et du travail à réaliser peuvent faire référence seulement aux situations normales considérées par les concepteurs. Ou ils peuvent comprendre une prise en compte de la variabilité, des incidents, des régulations que les opérateurs auront à mettre en œuvre. Par exemple, la simulation des zones d'atteinte sur une ligne d'assemblage peut être basée seulement sur le temps de cycle normal. Ou elle peut intégrer le fait que tous les cycles sont différents, et que compte tenu des incidents, les opérateurs peuvent couler ou remonter la chaîne. Ce qui conduira à des conclusions complètement différentes quant à l'espace nécessaire pour le poste de travail, probablement dans un rapport du simple au double.

Par conséquent, des modèles anthropométriques très sophistiqués ne sont en rien une garantie que le poste de travail sera conçu de façon appropriée, même d'un point de vue purement anthropométrique. L'enjeu critique est la mise en question de la description de la situation de travail et de l'activité qui s'y déroulera, les concepteurs n'étant pas en situation de décrire comment les personnes travailleront effectivement.

Si l'utilisation par les concepteurs de simulations basiques peut éviter des erreurs de conception graves (par exemple en permettant une prise en compte de la diversité anthropométrique), des outils techniques trop sophistiqués peuvent contribuer à l'illusion d'une « conception ergonomique » des situations de travail, alors que le seul travail qui a été pris en compte est la représentation qu'en ont les concepteurs. Une analyse préalable des déterminants du travail et des stratégies mises en œuvre par les opérateurs est évidemment essentielle.

\section{2.-Intérêts et limites des simulations expérimentales}

La seconde catégorie de simulations comporte un dispositif expérimental, qui simule l'environnement de travail et la dynamique du process, y compris les effets des actions des utilisateurs. Un ensemble de tâches à réaliser constitue le scénario de l'expérience. Les actions du ou des sujets et la dynamique du process sont enregistrées. Les résultats sont interprétés par l'observateur (l'ergonome), et seront utilisés pour faire évoluer la conception.

Il existe une littérature importante sur la validité et la fidélité de tels simulateurs (Nyssen, \& De Keyser, 1998). Leur principal intérêt est de permettre l'analyse de certaines caractéristiques de l'activité des utilisateurs dans des situations dont on espère qu'elles n'arriveront jamais dans la réalité.

On peut s'arrêter sur le statut des « sujets » quand une telle expérience fait partie du processus de conception. Laissons de côté les cas où les sujets sont des étudiants, des personnes recrutées spécifiquement pour l'expérience, ou des employés dont le travail n'a rien à voir avec celui qui est simulé, pour nous limiter au cas où les sujets ont des caractéristiques voisines de celles des futurs utilisateurs 
(Reuzeau, 2001).

Dans certains cas, les sujets sont placés dans un contexte expérimental, où leur comportement est analysé et interprété, sans qu'ils soient censés influencer de quelque façon le scénario de l'expérience. Le modèle du travail à réaliser est supposé indiscutable.

Dans d'autres cas, les sujets sont invités à influencer le déroulement de l'expérience, par exemple en suggérant de nouveaux scénarios à simuler. Cette contribution peut aussi être celle de l'instructeur, qui conduit parfois le simulateur et introduit parfois des événements imprévus qu'il/elle juge plausibles. Dans ces cas, les sujets ou l'instructeur sont considérés comme des partenaires de la conception. Cette configuration possède quelques-unes, mais pas l'ensemble des propriétés des simulations participatives que nous allons examiner maintenant.

\section{2.- Les simulations comme composantes d'une démarche participative}

Dans les interventions ergonomiques participatives, des groupes de travailleurs sont invités à participer à des simulations relatives au futur système de travail. La participation a été définie par Wilson (1991) comme l'implication des personnes dans la conception et le contrôle d'une part significative de leurs propres activités, avec suffisamment de connaissances et de pouvoir pour influencer à la fois les processus et les résultats, afin d'atteindre les buts souhaitables (traduit par nous).

Diverses formes d'organisation de la participation des travailleurs à la conception sont décrites dans la littérature. Certaines mettent l'accent sur la créativité des groupes de travail pour inventer des solutions satisfaisantes au début du processus de conception (par exemple les design decision groups, Wilson, 1991). D'autres sont organisées dans le temps pour correspondre à l'ensemble de la structure du processus de conception, et pour favoriser une confrontation continue entre les propositions des concepteurs et l'activité future possible correspondante (Daniellou, Garrigou, Kerguelen, \& Laville, 1990; Garrigou, Daniellou, Carballeda, \& Ruaud, 1995).

\section{1.-Les ingrédients de base d'une simulation participative}

Dans ce cas, des groupes de travailleurs sont constitués. Ils se réunissent régulièrement pendant le processus de conception. L'état d'avancement de la conception du système est examiné, et une simulation des conséquences sur l'activité future est réalisée. Un pronostic est établi, et conduit éventuellement à des modifications des décisions de conception.

Les composants de base d'une telle démarche sont:

- La construction sociale de l'ensemble du processus participatif;

- Le choix des participants;

- Le choix des supports de simulation et du type de simulation;

- Le choix des scénarios à simuler.

La construction sociale du processus doit permettre l'articulation entre les rôles de la maitrise d'ouvrage, de la maîtrise d'œuvre (Daniellou, 2004), des instances représentatives du personnel, et des groupes de travail. Les missions de chacun doivent être explicitées.

Le choix des participants dépend du type de connaissances professionnelles qui sont requises. Il peut être utile d'associer non seulement des opérateurs de production, mais aussi des opérateurs de maintenance, d'approvisionnement, de contrôle, etc., suivant les questions traitées. Un point-clé est la façon d'associer l'encadrement intermédiaire, soit dans les mêmes groupes soit dans des groupes spécifiques.

L'état de conception du système est représenté par des supports divers, plans, maquettes à échelle 
réduite ou grandeur nature, modèles informatiques, prototypes, etc., qui ont des propriétés différentes (Maline, 1994).

Suivant ces propriétés des supports utilisés, deux grands types de simulations sont possibles (Daniellou, 2005) : si l'on dispose d'un prototype, on peut effectuer une simulation expérimentale, où les sujets réalisent effectivement un certain nombre de tâches; dans le cas de plans ou de maquettes à échelle réduite, une simulation langagière est possible: les participants construisent un récit détaillé de modes opératoires possibles. Leur vraisemblance et les problèmes qu'ils engendrent sont discutés collectivement.

Le choix des scénarios est basé sur l'analyse préalable de situations de référence existantes, qui comportent certaines des sources de variabilité qui existeront dans le futur système (Daniellou, \& Garrigou, 1992). Ces scénarios sont préparés par l'ergonome, mais sont discutés et complétés par le groupe.

La simulation permet l'évaluation de risques pour la santé, de difficultés pour réaliser la qualité attendue, d'erreurs probables, etc. Les problèmes mineurs qui sont détectés peuvent être corrigés en temps réel, quand le chef de projet est présent, alors que des problèmes plus graves nécessiteront un complément d'études ou de nouvelles négociations, dont les résultats donneront lieu à une nouvelle séance de simulation (ibid).

\section{2.-On ne peut pas prévoir l'activité future}

Le but des simulations est d'approcher l'activité future des utilisateurs, et d'identifier les problèmes susceptibles de se poser, en termes de santé ou d'efficacité. Mais il faut souligner un point théorique essentiel; l'activité réelle des utilisateurs futurs ne peut pas être prévue en détail. Personne ne peut dire ce qu'une certaine patiente fera dans quelques années dans le hall du futur hôpital. En revanche, les décisions de conception ouvrent et ferment des pans entiers à l'activité future: certaines formes d'activité seront possibles, d'autres empêchées.

Le but d'une simulation n'est pas de prescrire la bonne façon de réaliser les tâches. Il s'agit d'évaluer quelles sont les formes possibles de l'activité future, et si elles sont acceptables selon les critères de l'ergonomie. Il est possible que les utilisateurs réels inventent des stratégies qui n'ont pas été prévues lors des simulations. Mais la simulation doit vérifier que, dans toutes les circonstances prévisibles, il existe au moins un mode opératoire qui soit acceptable pour réaliser les tâches.

\section{3.-Résultats et effets de la simulation}

Le résultat le plus connu de ces simulations participatives est bien sûr qu'elles permettent de détecter à temps des propriétés du futur système de travail susceptibles de provoquer des risques ou des dysfonctionnements, et de les corriger à un stade précoce de la conception plutôt qu'au démarrage de l'installation.

Mais les simulations participatives produisent aussi d'autres résultats que ceux qui concernent la conception du système technique. Des effets importants peuvent se produire i) en termes de formation, ii) du fait de la « confrontation de différents mondes ».

\section{Bénéfices formatifs et développementaux}

Nous adoptons la distinction faite par Rabardel et Béguin (2005) entre artefact et instrument. L'instrument est une entité mixte, constituée de l'artefact et des schèmes d'utilisation développés par l'utilisateur. Ces schèmes sont des invariants organisateurs de son activité. Pour que l'artefact devienne un instrument, une genèse instrumentale est nécessaire, qui combine le développement des schèmes d'utilisation par l'utilisateur, et les modifications que celui-ci fait subir à l'artefact pour « le mettre à sa main ». 
Des simulations successives relatives à un système de travail sont un bon cadre pour initier cette genèse instrumentale. Les utilisateurs futurs explorent le fonctionnement du nouveau système, et commencent ainsi à développer des schèmes d'utilisation. En même temps, ils influencent la conception du système, contribuant dans une certaine mesure à son adaptation.

Les travailleurs qui ont participé aux simulations font habituellement preuve, au moment du démarrage, d'une maîtrise du nouveau système bien supérieure à celle de leurs collègues qui ont seulement bénéficié des programmes de formation habituels.

\section{La rencontre des mondes}

Des simulations qui rassemblent des personnes appartenant à différents mondes professionnels sont aussi l'occasion d'une « rencontre » entre ces mondes. Ces différents mondes qui vont se rencontrer peuvent par exemple être celui des concepteurs, celui des opérateurs de production, celui des ingénieurs de production, etc.

Chaque participant peut découvrir que, dans l'entreprise, existent différents points de vue qui ne considèrent pas la situation de travail sous le même angle, qui mettent l'accent uniquement sur certaines des contraintes qui se trouvent rassemblées dans la situation de travail, et qui ne valorisent pas les mêmes aspects du travail qui s'y déroule. C'est aussi l'occasion de réaliser que ces différences ne sont pas affaire de personnalité, qu'il n'y a pas de « bonnes » et de «mauvaises » interprétations, que toutes les logiques sont nécessaires pour assurer le fonctionnement du système, mais qu'elles ne sont pas spontanément compatibles. La confrontation ouvre sur des débats qui peuvent conduire à des interprétations partagées des déterminants du travail.

Ces différences de points de vue peuvent, en première approche, être prises comme des différences de représentations de la situation de travail, ou d' « orientations sociocognitives » des participants (Garrigou et al., 1995). Mais Béguin (2005) souligne qu'elles sont plus que cela. Chaque catégorie professionnelle ne se contente pas de percevoir la situation de travail selon sa propre perspective. Elle agit de façon à maintenir la dynamique du process à l'intérieur de son « monde », c'est-à-dire dans les limites de ce qu'elle est capable de gérer.

Les simulations peuvent révéler que des classes de situations sont facilement gérées dans un «monde » ou un autre, tandis que d'autres sont susceptibles de ne l'être dans aucun des « mondes ». Cette situation est fréquente dans des industries chimiques, où la simulation peut porter sur des situations qui ne sont aisément gérées ni dans le monde de la conduite quotidienne, ni dans celui de l'ingénierie et du calcul. De tels constats mettent en évidence un «monde commun » de thèmes à explorer (Béguin, \& Clot, 2004; Béguin, 2005).

Ces confrontations et ces découvertes peuvent conduire à une modification du tissu social de l'organisation, et des relations entre groupes professionnels.

\section{3.- Discussion}

La simulation des postes de travail futurs apparaît comme un révélateur dans les débats théoriques sur la nature de l'ergonomie et sa contribution à l'amélioration des conditions de travail.

Une position consiste à considérer l'ergonomie comme une technologie qui applique des connaissances fondamentales sur la physiologie et la cognition humaines pour améliorer la conception des situations de travail. De ce point de vue, le travail n'est pas une question. Tout concepteur, tout ergonome sait en quoi consiste le travail, et peut lui appliquer l'expertise dont il est détenteur du fait de sa formation professionnelle. Chacun peut tranquillement utiliser des outils techniques de simulation pour évaluer un projet de conception, sans avoir à s'interroger sur sa propre représentation du travail futur. 
Une autre position est de considérer que l'ergonomie est une pratique (Daniellou, 1999), qui cherche à favoriser des débats, des processus de conception, et des décisions qui prennent en compte la complexité de ce qui se joue dans les situations de travail. De ce point de vue, l'activité n'est pas la simple exécution des tâches prescrites. Elle implique de faire face à la variabilité imprévue, de mobiliser des ressources individuelles et collectives, d'être impliquée dans des contradictions et des débats de valeurs. Elle comporte des coûts personnels et des tensions sociales. L'ergonome a un rôle dans la compréhension de cette densité de l'activité de travail, et dans l'ouverture de nouvelles voies pour son développement.

Comme l'indique Wilson (2000) : "Dans ce rôle, nous sommes des artisans, qui utilisons notre jugement, notre vision, notre expérience, et parfois des essais et erreurs pour développer et mettre à l'épreuve des concepts et des prototypes » (traduit par nous).

Si l'on accepte cette position, l'ergonomie se doit de renforcer ses interfaces non seulement avec la physiologie et la psychologie cognitive, mais avec les sciences sociales et la psychologie du développement. Et les ergonomes ont besoin de débattre des modèles qu'ils mettent en œuvre, non seulement sur l'être humain au travail, mais aussi sur leur propre activité (Daniellou, 1999).

\section{RÉFÉRENCEMENT}

Daniellou, F. (2007). Des fonctions de la simulation des situations de travail en ergonomie. @ctivités, 4 (2), pp. 77-83, http://www.activites.org/v4n2/v4n2.pdf

\section{BibLIOGRAPHIE}

Béguin, P. (2005). Concevoir pour les genèses professionnelles. In P. Rabardel, \& P. Pastré (Eds.), Modèles du sujet pour la conception (pp. 31-52). Toulouse: Octarès Editions.

Béguin, P, \& Clot, Y. (2004). Situated action in the development of activity. @ctivités, 2004, 1 (2), 50-63, http://www.activites.org/v1n2/beguin.eng.pdf

Daniellou, F., (1999). The ergonomist is a worker? That is the (epistemological) question. In N. Marmaras (Ed.), Strengths and weaknesses, threats and opportunities of ergonomics in front of 2000. Athens: The Hellenic ergonomics society.

Daniellou, F., (2004). L'ergonomie dans la conduite de projets de conception de systèmes de travail. In P. Falzon (Ed.), Traité d'ergonomie (pp. 359-373). Paris: PUF

Daniellou, F. (2005). How far can future activity be foreseen ? Proceedings of First ISCAR Congress, Acting in changing worlds, Universidad de Sevilla and Universidad Pablo Olavide de Sevilla.

Garrigou, A., Daniellou, F., Carballeda, G., \& Ruaud, S. (1995). Activity analysis in participatory design and analysis of participatory design activity. International Journal of Industrial Ergonomics, 15 (5), 311-327.

Daniellou, F., \& Garrigou, A. (1992). Human Factors in design: sociotechnics or ergonomics. In M. Helander, \& M. Nagamachi (Eds.), Design for manufacturability (pp. 53-63). London: Taylor and Francis.

Daniellou, F., Garrigou, A., Kerguelen, A., \& Laville, A. (1990). Taking future activity in account at the design stage : participative design in the printing industry. In C.H. Haslegrave, J.R.Wilson, E.N. Corlett, \& I. Manenica (Eds.), Work design in practice, Proceedings of the Third. International Occupational Ergonomics Symposium Zadar (pp. 189-196). London: Taylor and Francis.

Haslegrave, C., Wilson, J.R., \& Corlett E.N. (1990). Work design in practice. Proceedings of the $3^{\text {rd }}$ International Occupational Ergonomics Symposium Zadar. London: Taylor and Francis.

Maline, J. (1994). Simuler le travail : une aide à la conduite de projet. Lyon: Anact (Outils et Méthodes).

Nyssen, A.S., \& De Keyser, V. (1998). Improving training in problem solving skills: analysis of anesthetist's performance in simulated problem situations. Le Travail Humain, 61 (4), 387-401.

Pastré, P. (2005). Apprendre par la simulation. Toulouse: Octarès Editions. 
Rabardel, P., \& Béguin, P. (2005). Instrument mediated activity : from subject development to anthropocentric design. Theoretical Issues in Ergonomics Science, 6 (5), 429-461.

Reuzeau, F. (2001). Finding the best users to involve in design : a rational approach. Le Travail Humain, 64 (3), 223-245.

Wilson, J.R. (1991). Ergonomics and participation. In: J.R. Wilson, \& E.N. Corlett (Eds.), Evaluation of Human Work (pp. 1071-1096). London: Taylor and Francis.

Wilson, J.R., (2000). Fundamentals of ergonomics in theory and practice. Applied ergonomics, 31, 557-567.

\section{RÉSUMÉ}

On peut catégoriser les simulations ergonomiques selon la place qu'elles attribuent aux futurs utilisateurs : ceux-ci peuvent être modélisés par des dispositifs techniques, être sujets d'une expérimentation, ou être impliqués dans un processus participatif. Dans ce dernier cas, les simulations sont susceptibles de produire des effets qui dépassent les améliorations techniques de conception, et déboucher sur de nouveaux développements de l'activité individuelle et collective.

\section{MoTS CLÉS}

Simulation, ergonomie participative, théorie de la pratique

\section{RESUMEN}

Las simulaciones ergonómicas se pueden categorizar de acuerdo al lugar que éstas le asignan a los futuros usuarios: éstos pueden ser modelizados mediante dispositivos técnicos, ser sujetos de una experimentación, o estar implicados en un proceso participativo. En este último caso, las simulaciones son susceptibles de producir efectos que sobrepasan las mejoras técnicas de diseño, y desembocar en nuevos desarrollos de la actividad individual y colectiva.

Palabras Clave

Simulación, ergonomía participativa, teoría de la práctica 